\title{
Nucleon Vector and Axial-Vector Form Factors
}

\section{Yong-Chull Jang*, Tanmoy Bhattacharya, Rajan Gupta}

Theoretical Division, T-2, Los Alamos National Laboratory, Los Alamos, NM, 87545, U.S.A.

E-mail: ypjelanl.gov

\section{Boram Yoon}

Computer, Computational, and Statistical Sciences, CCS-7, Los Alamos National Laboratory, Los Alamos, NM, 87545, U.S.A.

\section{Huey-Wen Lin}

Department of Physics and Astronomy, Michigan State University, MI, 48824, U.S.A

\section{PNDME Collaboration ${ }^{\dagger}$}

We present a status report on the calculation of the isovector axial-vector and vector form factors of the nucleon using (i) eight ensembles of $N_{f}=2+1+1$ HISQ fermions at lattice spacings $a=0.12,0.09$, and $0.06 \mathrm{fm}$ and three values of light quark masses corresponding to $M_{\pi}=310,220,130 \mathrm{MeV}$ generated by the MILC collaboration and (ii) four ensembles of clover fermions generated by the JLAB/W\&M collaboration. In both cases, the clover action is used for valence quarks. Analysis of the $Q^{2}$ dependence of the form factors is carried out using the $z$-expansion and the dipole ansatz. The results for the charge radii and dipole masses are compared and found to be consistent between the two fit ansatz and between the two lattice formulations.

34th annual International Symposium on Lattice Field Theory

24-30 July 2016

University of Southampton, UK

\footnotetext{
${ }^{*}$ Speaker.

${ }^{\dagger}$ The calculation of the form factors on the 2+1-clover ensembles is being done in collaboration with Balint Joo, Kostas Orginos, David Richards and Frank Winter.
} 


\section{Introduction}

The isovector vector and axial-vector form factors of the nucleon are measured in a number of experiments and important properties such as the electric $\left(r_{E}\right)$ and magnetic $\left(r_{M}\right)$ charge radii of the proton have been extracted from electron scattering and [muonic] hydrogen spectrum. At present, there is a roughly $7 \sigma$ difference between $r_{E}=0.8751(61)$ fm from the CODATA world average using electron scattering data [1] and $r_{E}=0.84087(39)$ fm from Lamb shift in muonic hydrogen [2,3]. The proton magnetic radius is $r_{M}=0.86_{-0.03}^{+0.02} \mathrm{fm} \mathrm{[4];} \mathrm{the} \mathrm{magnetic} \mathrm{moment} \mathrm{is} \mu^{p}=$ 2.792847356(23) for proton and $\mu^{n}=1.9130427(5)$ for neutron [5]. The axial charge radius is $r_{A}=0.80_{-0.17}^{+0.07} \pm 0.12 \mathrm{fm}$ (neutrino scattering) [6], $r_{A}=0.74_{-0.09}^{+0.12} \pm 0.05 \mathrm{fm}$ (electroproduction) [6], $r_{A}=0.68(16) \mathrm{fm}$ (deuterium target) [7]. The goal of lattice calculations is to reach this level of precision and help resolve the various discrepencies.

The axial form-factors of nucleons are important input in the calculation of the cross-section of neutrinos on nuclear targets. These energy dependent cross-sections are needed to determine the neutrino flux, a major systematic in neutrino oscillation experiments. There is, at present, a $3 \sigma$ tension between different phenomenological estimates of the effective axial mass extracted using the dipole approximation to fit the data (see Ref. [7]).

Lack of total control over systematics in vector and axial-vector form factors afflict both experimental analyses and lattice calculations. The goal is to continuously increase the precision of both experiments and lattice calculations to establish agreement or to invesigate beyond the standard model contributions in case of disagreement. In this talk we summarize the status of lattice calculations using two lattice formulations: 8 ensembles with clover-on-HISQ analysis and 4 ensembles of clover-on-clover. The lattice parameters of these ensembles are described in Refs. $[8,9,10]$.

\section{Nucleon Form Factors}

The matrix element of the vector current within the nucleon state can be decomposed in terms of the Dirac, $F_{1}$, and Pauli, $F_{2}$, form factors as:

$$
\left\langle N\left(\boldsymbol{p}_{f}\right)\left|V_{\mu}(\boldsymbol{Q})\right| N\left(\boldsymbol{p}_{i}\right)\right\rangle=\bar{u}_{N}\left(\boldsymbol{p}_{f}\right)\left[\gamma_{\mu} F_{1}\left(Q^{2}\right)+\sigma_{\mu v} Q_{\mu} \frac{F_{2}\left(Q^{2}\right)}{2 m_{N}}\right] u_{N}\left(\boldsymbol{p}_{i}\right),
$$

where the four-momentum transfer $Q_{\mu} \equiv\left(p_{f}-p_{i}\right)_{\mu}$ and $Q^{2}=\boldsymbol{p}_{i}^{2}-\left(m_{N}-E_{i}\right)^{2}$. The momentum $\boldsymbol{p}_{f} \equiv 0$ since we insert a zero-momentum nucleon state at the sink timeslice. The experimental differential cross section is commonly written in terms of the Sachs electric, $G_{E}$, and magnetic, $G_{M}$, form factors defined as [11]:

$$
G_{E}\left(Q^{2}\right)=F_{1}\left(Q^{2}\right)-\frac{Q^{2}}{4 m_{N}^{2}} F_{2}\left(Q^{2}\right), \quad G_{M}\left(Q^{2}\right)=F_{1}\left(Q^{2}\right)+F_{2}\left(Q^{2}\right) .
$$

The axial form factor, $G_{A}$, and the induced pseudoscalar form factor, $G_{P}$, are obtained from the matrix element of the axial-vector current within the nucleon state:

$$
\left\langle N\left(\boldsymbol{p}_{f}\right)\left|A_{\mu}(\boldsymbol{Q})\right| N\left(\boldsymbol{p}_{i}\right)\right\rangle=\bar{u}_{N}\left(\boldsymbol{p}_{f}\right)\left[\gamma_{\mu} G_{A}\left(Q^{2}\right)+Q_{\mu} \frac{G_{P}\left(Q^{2}\right)}{2 m_{N}}\right] \gamma_{5} u_{N}\left(\boldsymbol{p}_{i}\right)
$$




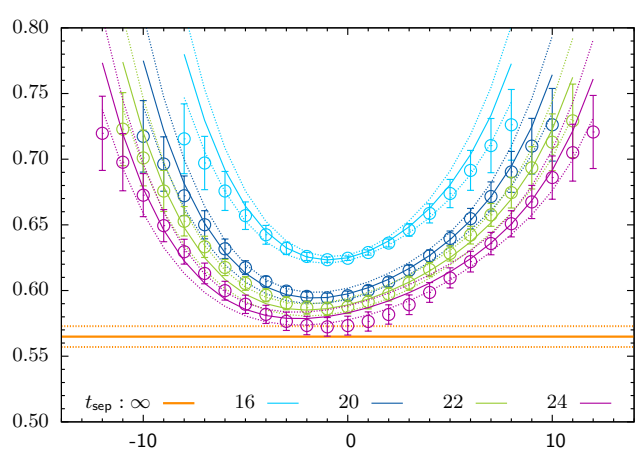

(a) 2-state

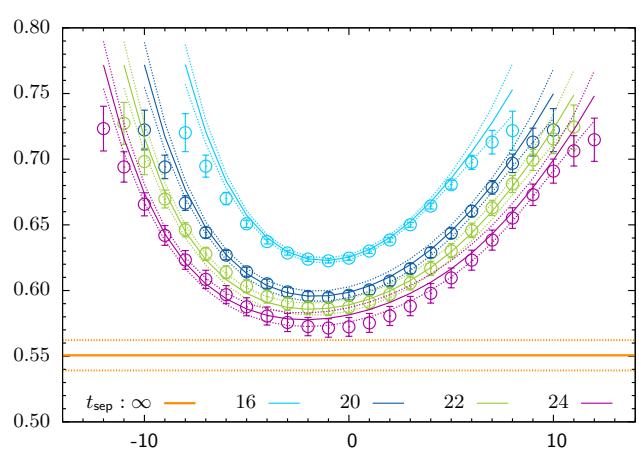

(b) 4-state

Figure 1: Data for the 3-point correlator ratio, Eq. (3.3), from the a06m220 HISQ ensemble with $V_{4}$ operator insertion at lattice momenta $(1,1,1) . G_{E}\left(Q^{2}\right)$ are extracted from such data.

On the lattice, one first calculates nucleon 3-point functions with vector and axial-vector operator insertion for a large set of momentum transfer $Q_{\mu}$. From these 3-point functions, we extract matrix elements as described in $[9,10]$. Various form factors are obtained from linear combinations of these matrix elements using appropriate Dirac matrices $\gamma_{\mu}$ and values of momentum insertion $Q_{\mu}$.

\section{Controlling the Excited-State Contamination (ESC)}

High statistics estimates were obtained cost-effectively by using the truncated solver method with bias correction (AMA) [12, 13], and the coherent source sequential propagator method [9, 10]. The tuning of the covariant gaussian source smearing size $\sigma$ and the values of the source-sink separations $t_{\text {sep }}$ used in the simulations are described in Refs. $[9,10]$. Results presented here are obtained using amplitudes $\mathscr{A}_{i}$ and energies $E_{i}$ from 4-state fits to 2-point correlators, stablized using non-trivial priors as described in Ref. [10, 8]:

$$
C^{(2 \mathrm{pt})}(t, \boldsymbol{p})=\left|\mathscr{A}_{0}\right|^{2} e^{-E_{0} t}+\left|\mathscr{A}_{1}\right|^{2} e^{-E_{1} t}+\left|\mathscr{A}_{2}\right|^{2} e^{-E_{2} t}+\left|\mathscr{A}_{3}\right|^{2} e^{-E_{3} t} .
$$

These $\mathscr{A}_{i}$ and $E_{i}$ are used as inputs in 2-state fits to 3-point data at multiple $t_{\text {sep }}$ and $\tau$ to extract the matrix element $\left\langle 0^{\prime}\left|\mathscr{O}_{\Gamma}\right| 0\right\rangle$ in the $t_{\text {sep }} \rightarrow \infty$ limit:

$$
\begin{aligned}
C_{\Gamma}^{(3 \mathrm{pt})}\left(t ; \tau ; \boldsymbol{p}^{\prime}, \boldsymbol{p}\right) & =\left|\mathscr{A}_{0}^{\prime}\right|\left|\mathscr{A}_{0}\right|\left\langle 0^{\prime}\left|\mathscr{O}_{\Gamma}\right| 0\right\rangle e^{-E_{0} t-M_{0}(\tau-t)}+\left|\mathscr{A}_{1}^{\prime}\right|\left|\mathscr{A}_{1}\right|\left\langle 1^{\prime}\left|\mathscr{O}_{\Gamma}\right| 1\right\rangle e^{-E_{1} t-M_{1}(\tau-t)} \\
& +\left|\mathscr{A}_{0}^{\prime}\right|\left|\mathscr{A}_{1}\right|\left\langle 0^{\prime}\left|\mathscr{O}_{\Gamma}\right| 1\right\rangle e^{-E_{0} t-M_{1}(\tau-t)}+\left|\mathscr{A}_{1}^{\prime}\right|\left|\mathscr{A}_{0}\right|\left\langle 1^{\prime}\left|\mathscr{O}_{\Gamma}\right| 0\right\rangle e^{-E_{1} t-M_{0}(\tau-t)} .
\end{aligned}
$$

To estimate errors, these two fits are done within a single Jackknife process with the $\chi^{2} /$ d.o.f. for each fit minimized using the full covariance matrix. The charges $G_{A}$ and $G_{E}$ used for normalizing the form factors are taken from 3 -state fits to the 3-point correlators [10, 8].

In Fig. 1, we illustrate ESC in 3-point functions by plotting the ratio, Eq. (3.3), with the insertion of the $V_{4}$ operator at lattice momentum $(1,1,1)$. Dependence on $t_{\text {sep }}$ and $\tau$ indicates ESC. We control this by making combined fits versus $t_{\text {sep }}$ and $\tau$ using Eq. 3.2. The input amplitudes and energies, $\mathscr{A}_{i}$ and $E_{i}$, are from 2-state (Fig. 1a) and 4-state (Fig. 1b) fits to Eq. (3.1).

$$
\frac{C_{\Gamma}^{(3 \mathrm{pt})}\left(t, \tau ; \boldsymbol{p}^{\prime}, \boldsymbol{p}\right)}{C^{(2 \mathrm{pt})}\left(\tau, \boldsymbol{p}^{\prime}\right)}\left[\frac{C^{(2 \mathrm{pt})}\left(t, \boldsymbol{p}^{\prime}\right) C^{(2 \mathrm{pt})}\left(\tau, \boldsymbol{p}^{\prime}\right) C^{(2 \mathrm{pt})}(\tau-t, \boldsymbol{p})}{C^{(2 \mathrm{pt})}(t, \boldsymbol{p}) C^{(2 \mathrm{pt})}(\tau, \boldsymbol{p}) C^{(2 \mathrm{pt})}\left(\tau-t, \boldsymbol{p}^{\prime}\right)}\right]^{1 / 2} .
$$




\begin{tabular}{c|c|ccccccc}
\hline \hline Ensemble & Fit & $r_{E}[\mathrm{fm}]$ & $\chi^{2} / \mathrm{DOF}$ & $r_{M}[\mathrm{fm}]$ & $\mu$ & $\chi^{2} / \mathrm{DOF}$ & $r_{A}[\mathrm{fm}]$ & $\chi^{2} / \mathrm{DOF}$ \\
\hline \multirow{3}{*}{ a12m310AMA } & $\mathrm{D}$ & $0.729(021)$ & 1.026 & $0.643(028)$ & $4.234(057)$ & 0.664 & $0.473(022)$ & 1.150 \\
& $z^{2}$ & $0.742(007)$ & 1.630 & $0.694(015)$ & $4.370(078)$ & 0.448 & $0.494(011)$ & 0.709 \\
& $z^{3}$ & $0.731(015)$ & 2.132 & $0.641(109)$ & $4.250(241)$ & 0.620 & $0.481(026)$ & 0.896 \\
\hline \multirow{3}{*}{ a12m220LAMA } & $\mathrm{D}$ & $0.764(034)$ & 0.234 & $0.683(068)$ & $4.252(098)$ & 0.721 & $0.498(053)$ & 0.392 \\
& $z^{2}$ & $0.791(016)$ & 0.130 & $0.750(039)$ & $4.370(130)$ & 0.198 & $0.511(023)$ & 0.423 \\
& $z^{3}$ & $0.791(049)$ & 0.194 & $0.811(115)$ & $4.439(184)$ & 0.118 & $0.517(038)$ & 0.613 \\
\hline \multirow{3}{*}{ 099m310 } & $\mathrm{D}$ & $0.677(056)$ & 1.005 & $0.648(103)$ & $4.146(131)$ & 0.487 & $0.487(091)$ & 0.263 \\
& $z^{2}$ & $0.692(021)$ & 1.255 & $0.683(034)$ & $4.227(161)$ & 0.884 & $0.496(030)$ & 0.353 \\
& $z^{3}$ & $0.718(053)$ & 1.755 & $0.359(424)$ & $3.705(432)$ & 0.070 & $0.479(049)$ & 0.432 \\
\hline \multirow{4}{*}{ a09m220 } & $\mathrm{D}$ & $0.719(083)$ & 0.318 & $0.674(126)$ & $4.046(148)$ & 0.941 & $0.492(116)$ & 1.169 \\
& $z^{2}$ & $0.730(037)$ & 0.538 & $0.728(074)$ & $4.105(193)$ & 1.651 & $0.524(057)$ & 1.564 \\
& $z^{3}$ & $0.613(140)$ & 0.344 & - & - & - & $0.613(079)$ & 1.476 \\
\hline \multirow{3}{*}{ 006m310AMA } & $\mathrm{D}$ & $0.914(076)$ & 7.356 & $0.712(098)$ & $3.483(086)$ & 1.798 & $0.594(100)$ & 6.206 \\
& $z^{2}$ & $1.101(044)$ & 5.061 & $0.258(446)$ & $3.301(129)$ & 1.388 & $0.664(040)$ & 8.146 \\
& $z^{3}$ & $1.341(068)$ & 0.250 & $1.338(365)$ & $3.874(399)$ & 0.474 & $0.937(076)$ & 6.769 \\
\hline \multirow{3}{*}{ a06m220AMA } & $\mathrm{D}$ & $0.708(074)$ & 0.692 & $0.648(092)$ & $3.987(100)$ & 0.974 & $0.495(094)$ & 0.782 \\
& $z^{2}$ & $0.712(023)$ & 0.763 & $0.652(029)$ & $3.954(109)$ & 0.014 & $0.496(027)$ & 0.240 \\
& $z^{3}$ & $0.755(038)$ & 0.218 & $0.643(155)$ & $3.935(341)$ & 0.024 & $0.493(039)$ & 0.354 \\
\hline & $\mathrm{D}$ & $0.741(039)$ & 2.165 & $0.643(053)$ & $3.821(063)$ & 0.706 & $0.484(046)$ & 0.788 \\
& $z^{2}$ & $0.778(016)$ & 1.227 & $0.695(035)$ & $3.891(090)$ & 0.728 & $0.492(020)$ & 0.925 \\
& $z^{3}$ & $0.812(028)$ & 0.834 & $0.856(153)$ & $4.128(275)$ & 0.628 & $0.519(038)$ & 1.037 \\
\hline & $\mathrm{D}$ & $0.752(073)$ & 0.899 & $0.667(091)$ & $3.916(132)$ & 0.678 & $0.464(079)$ & 0.719 \\
& $z^{2}$ & $0.817(057)$ & 0.887 & $0.641(150)$ & $3.915(200)$ & 0.787 & $0.395(083)$ & 0.777 \\
& $z^{3}$ & $0.820(116)$ & 1.034 & $0.560(744)$ & $3.873(400)$ & 0.940 & $0.378(197)$ & 0.904 \\
\hline
\end{tabular}

Table 1: Charge radii from the Clover-on-HISQ analysis

\section{The Nucleon Charge Radii}

The mean squared charge radii $r_{i}(i \in$ electric $(E)$, magnetic $(M)$ and axial $(A))$, are defined by the slope of the form factor with respect to the momentum transfer $Q^{2}$ at $Q^{2}=0$ :

$$
\left\langle r_{i}^{2}\right\rangle \equiv-\left.6 \frac{d}{d Q^{2}}\left(\hat{G}_{i}\left(Q^{2}\right) / \hat{G}_{i}(0)\right)\right|_{Q^{2}=0} .
$$

We use the normalized $\hat{G}_{E, M}=G_{E, M} / g_{E}$ and $\hat{G}_{A, P}=G_{A, P} / g_{A}$ with $g_{E}$ the electric charge and $g_{A}$ the axial charge. $\hat{G}_{E, M}\left(Q^{2}\right)$ and $\hat{G}_{A}\left(Q^{2}\right)$ are commonly parameterized using the dipole ansatz,

$$
\hat{G}_{i}\left(Q^{2}\right)=\hat{G}_{i}(0) /\left(1+Q^{2} / \mathscr{M}_{i}^{2}\right)^{2},
$$

where $\mathscr{M}_{i}$ is the dipole mass, in terms of which the charge radii are $\left\langle r_{i}^{2}\right\rangle=12 / \mathscr{M}_{i}^{2}$. By construction $\hat{G}_{E, A}(0)=1$ and the anomalous magnetic moment $\kappa$ is $\hat{G}_{M}(0)=\mu \equiv 1+\kappa$. The dipole fits for $G_{E}$, $G_{M}$, and $G_{A}$ are shown in Figs. 2 and 3 and estimates of the radii in Tables 1 and 2.

Experimental data are often analyzed using the Kelly parameterization [14]:

$$
\hat{G}_{X}\left(Q^{2}\right)=\hat{G}(0) \sum_{k=0}^{n} a_{k} \tau^{k} /\left\{1+\sum_{k=1}^{n+2} b_{k} \tau^{k}\right\}, \quad \hat{G}_{Y}\left(Q^{2}\right)=\frac{A \tau}{1+B \tau} \frac{1}{\left(1+Q^{2} / 0.71 \mathrm{GeV}^{2}\right)^{2}},
$$

where $\tau=Q^{2} / 4 \mathscr{M}^{2}$. The parameters $\mathscr{M}, G(0), a_{k}, b_{k}, A$, and $B$ are determined from fit to the data. The parameterization $\hat{G}_{X}\left(Q^{2}\right)$ is used for the electic and magentic form factors for the proton and 


\begin{tabular}{c|c|ccccccc}
\hline \hline Ensemble & Fit & $r_{E}[\mathrm{fm}]$ & $\chi^{2} / \mathrm{DOF}$ & $r_{M}[\mathrm{fm}]$ & $\mu$ & $\chi^{2} / \mathrm{DOF}$ & $r_{A}[\mathrm{fm}]$ & $\chi^{2} / \mathrm{DOF}$ \\
\hline \multirow{3}{*}{$\mathrm{a} 114 \mathrm{~m} 315\left(S_{5} S_{5}\right)$} & $\mathrm{D}$ & $0.770(030)$ & 0.417 & $0.667(033)$ & $4.384(043)$ & 0.239 & $0.488(028)$ & 0.882 \\
& $z^{2}$ & $0.769(009)$ & 0.544 & $0.690(010)$ & $4.428(044)$ & 0.346 & $0.501(009)$ & 0.559 \\
& $z^{3}$ & $0.781(012)$ & 0.227 & $0.680(019)$ & $4.408(054)$ & 0.335 & $0.500(012)$ & 0.652 \\
\hline \multirow{3}{*}{$\mathrm{a} 081 \mathrm{~m} 315\left(S_{5} S_{5}\right)$} & $\mathrm{D}$ & $0.715(057)$ & 0.871 & $0.616(086)$ & $4.168(136)$ & 1.366 & $0.439(075)$ & 1.325 \\
& $z^{2}$ & $0.717(017)$ & 0.909 & $0.690(018)$ & $4.432(131)$ & 2.037 & $0.482(021)$ & 2.195 \\
& $z^{3}$ & $0.702(041)$ & 1.031 & $0.479(107)$ & $3.876(229)$ & 0.704 & $0.412(037)$ & 1.329 \\
\hline & $\mathrm{D}$ & $0.704(039)$ & 0.577 & $0.596(067)$ & $4.201(108)$ & 1.044 & $0.445(081)$ & 0.749 \\
$\mathrm{a} 081 \mathrm{~m} 315\left(S_{7} S_{7}\right)$ & $z^{2}$ & $0.716(010)$ & 0.443 & $0.677(021)$ & $4.460(127)$ & 1.270 & $0.480(024)$ & 1.053 \\
& $z^{3}$ & $0.689(023)$ & 0.238 & $0.600(082)$ & $4.228(244)$ & 1.275 & $0.438(035)$ & 0.582 \\
\hline & $\mathrm{D}$ & $0.703(022)$ & 1.874 & $0.593(035)$ & $4.108(087)$ & 1.459 & $0.424(026)$ & 2.059 \\
$\mathrm{a} 081 \mathrm{~m} 315\left(S_{9} S_{9}\right)$ & $z^{2}$ & $0.709(006)$ & 0.656 & $0.671(013)$ & $4.368(106)$ & 0.831 & $0.462(011)$ & 1.255 \\
& $z^{3}$ & $0.716(019)$ & 0.741 & $0.658(064)$ & $4.327(220)$ & 0.987 & $0.466(023)$ & 1.454 \\
\hline \multirow{3}{*}{$\mathrm{a} 079 \mathrm{~m} 195\left(S_{7} S_{7}\right)$} & $\mathrm{D}$ & $0.726(039)$ & 0.648 & $0.638(044)$ & $4.105(079)$ & 1.064 & $0.453(037)$ & 0.417 \\
& $z^{2}$ & $0.767(018)$ & 0.621 & $0.705(043)$ & $4.195(120)$ & 1.396 & $0.453(023)$ & 0.519 \\
& $z^{3}$ & $0.746(030)$ & 0.600 & $0.472(275)$ & $3.949(243)$ & 1.404 & $0.423(042)$ & 0.466 \\
\hline \multirow{3}{*}{$\mathrm{a} 079 \mathrm{~m} 195 \mathrm{~L}\left(S_{7} S_{7}\right)$} & $\mathrm{D}$ & $0.750(050)$ & 1.379 & $0.707(068)$ & $4.514(119)$ & 0.485 & $0.546(078)$ & 0.473 \\
& $z^{2}$ & $0.780(033)$ & 1.645 & $0.753(037)$ & $4.555(130)$ & 0.866 & $0.566(032)$ & 0.503 \\
& $z^{3}$ & $0.743(055)$ & 1.788 & $0.587(142)$ & $4.404(165)$ & 0.594 & $0.577(048)$ & 0.572 \\
\hline \hline
\end{tabular}

Table 2: Charge radii from the Clover-on-Clover analysis

\begin{tabular}{l|ccc}
\hline \hline \multicolumn{1}{c|}{ Ensemble } & $\mathscr{M}_{E}[\mathrm{GeV}]$ & $\mathscr{M}_{M}[\mathrm{GeV}]$ & $\mathscr{M}_{A}[\mathrm{GeV}]$ \\
\hline a12m310AMA & $0.938(008)$ & $1.062(013)$ & $1.444(020)$ \\
\hline a12m220LAMA & $0.895(012)$ & $1.002(029)$ & $1.372(042)$ \\
\hline a09m310 & $1.010(024)$ & $1.055(048)$ & $1.405(076)$ \\
\hline a09m220 & $0.950(032)$ & $1.014(055)$ & $1.388(094)$ \\
\hline a09m130AMA & $0.748(018)$ & $0.961(038)$ & $1.151(056)$ \\
\hline a06m310AMA & $0.965(029)$ & $1.055(043)$ & $1.380(075)$ \\
\hline a06m220AMA & $0.923(014)$ & $1.063(025)$ & $1.413(039)$ \\
\hline a06m130AMA & $0.909(025)$ & $1.025(040)$ & $1.475(073)$ \\
\hline \hline
\end{tabular}

\begin{tabular}{l|ccc}
\hline \hline \multicolumn{1}{c|}{ Ensemble } & $\mathscr{M}_{E}[\mathrm{GeV}]$ & $\mathscr{M}_{M}[\mathrm{GeV}]$ & $\mathscr{M}_{A}[\mathrm{GeV}]$ \\
\hline $\mathrm{a} 114 \mathrm{~m} 315\left(S_{5} S_{5}\right)$ & $0.888(010)$ & $1.025(015)$ & $1.402(023)$ \\
\hline $\mathrm{a} 081 \mathrm{~m} 315\left(S_{5} S_{5}\right)$ & $0.956(022)$ & $1.110(045)$ & $1.556(077)$ \\
\hline $\mathrm{a} 081 \mathrm{~m} 315\left(S_{7} S_{7}\right)$ & $0.971(016)$ & $1.148(037)$ & $1.534(081)$ \\
\hline $\mathrm{a} 081 \mathrm{~m} 315\left(S_{9} S_{9}\right)$ & $0.973(009)$ & $1.152(020)$ & $1.612(029)$ \\
\hline $\mathrm{a} 079 \mathrm{~m} 195\left(S_{7} S_{7}\right)$ & $0.941(015)$ & $1.072(021)$ & $1.508(035)$ \\
\hline $\mathrm{a} 079 \mathrm{~m} 195 \mathrm{~L}\left(S_{7} S_{7}\right)$ & $0.912(018)$ & $0.967(027)$ & $1.252(052)$ \\
\hline \hline
\end{tabular}

(b) dipole masses from the Clover-on-Clover analysis

(a) dipole masses from the Clover-on-HISQ analysis

Table 3: Estimates of dipole mass

the magnetic form factor for the neutron, while the electric form factor for the neutron is fit using $G_{Y}$. The lattice data are compared against Kelly fits to experimental data in Fig. 2. For the Kelly fits shown in Fig. 2, we used the parameters given in Table I in Ref. [14].

A model independent approach, incorporating analyticity of QCD, is the $z$-expansion $[15,6]$ :

$$
\hat{G}\left(Q^{2}\right)=\sum_{k=0}^{\infty} a_{k} z\left(Q^{2}\right)^{k} \quad \text { with } \quad z=\frac{\sqrt{t_{\mathrm{cut}}+Q^{2}}-\sqrt{t_{\mathrm{cut}}+Q_{0}^{2}}}{\sqrt{t_{\mathrm{cut}}+Q^{2}}+\sqrt{t_{\mathrm{cut}}+Q_{0}^{2}}} .
$$

The $a_{k}$ are fit parameters. Using $t_{\text {cut }}=4 m_{\pi}^{2}$, position of the branch cut for the electric form factor [15] and $t_{\text {cut }}=9 m_{\pi}^{2}$ for the axial form factor [6], the domain of analyticity in $z$ is the unit circle. We set the free parameter $Q_{0}=0$, and for $m_{\pi}$ use the pion with sea quark mass for each ensemble. For the magnetic form factor, we use the same $t_{\text {cut }}$ as for the electic form factor. Results for the radii $r_{i}$, using a quadratic and cubic $z$-expansion, are also given in Tables 1 and 2.

Lastly, we compare in Figs. $3 \mathrm{c}$ and $3 \mathrm{~d}$ the lattice data for the pseudoscalar form factor $\hat{G}_{P}$ 


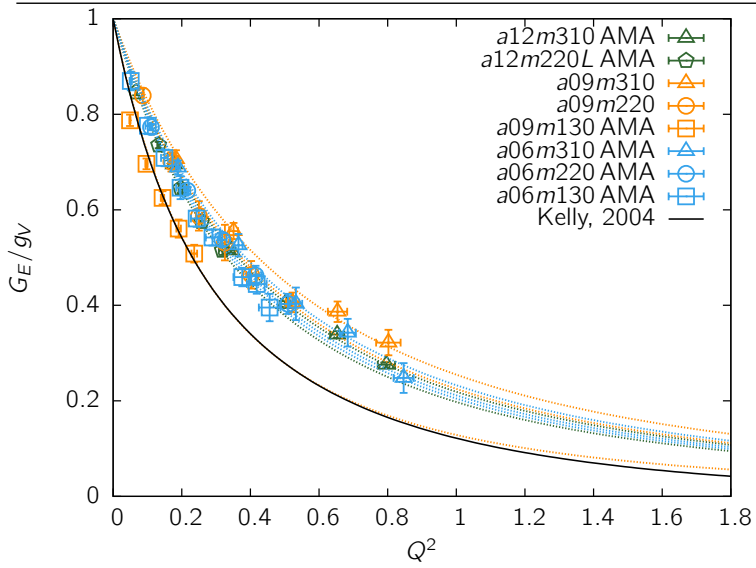

(a) $G_{E}$, Clover-on-HISQ analysis

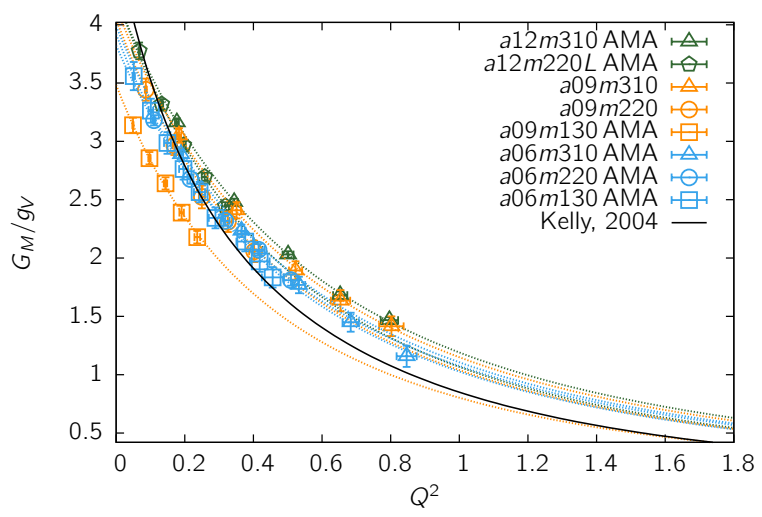

(c) $G_{M}$, Clover-on-HISQ analysis

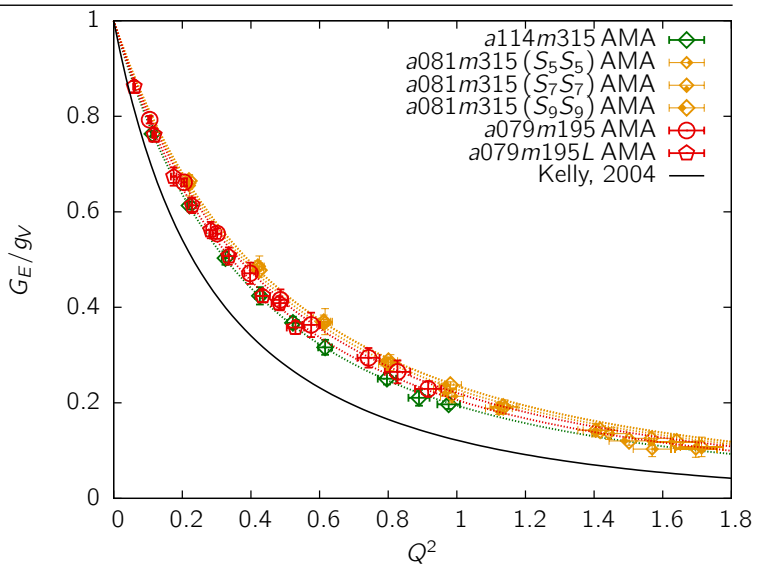

(b) $G_{E}$, Clover-on-Clover analysis

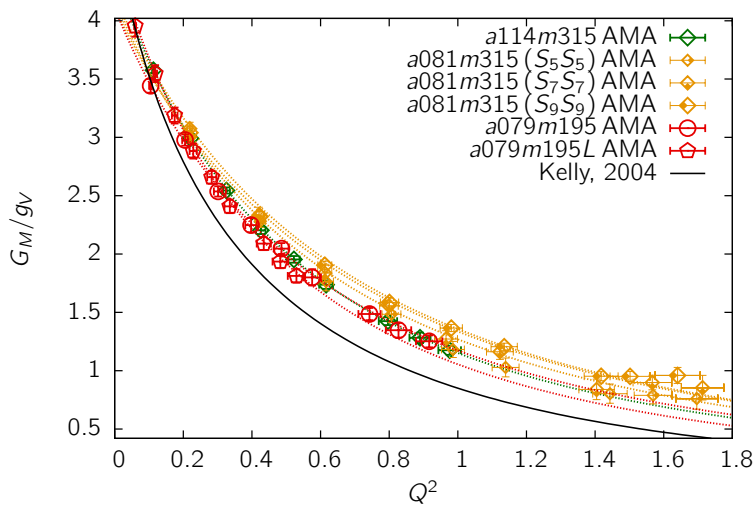

(d) $G_{M}$, Clover-on-Clover analysis

Figure 2: Data for $G_{E}\left(Q^{2}\right)$ and $G_{M}\left(Q^{2}\right)$ and dipole and Kelly fits to them.

against the pion-pole dominance ansatz (product of the axial form factor times a pion pole):

$$
\hat{G}_{P}\left(Q^{2}\right)=\hat{G}_{A}\left(Q^{2}\right)\left[\frac{4 m_{p}^{2}}{Q^{2}+m_{\pi}^{2}}\right],
$$

where we use $m_{p}=939 \mathrm{MeV}$ for the proton mass and $m_{\pi}=135 \mathrm{MeV}$ for the pion mass.

\section{Conclusions}

Some concluding observations are: (i) the dipole ansatz works well except on the a09m130 HISQ ensemble. (ii) Estimates of $r_{i}$ agree between the dipole and fits quadratic and cubic in $z$. (iii) The $r_{i}$ are slightly larger (i.e. $\mathscr{M}_{i}$ slightly smaller) from comparable clover-on-clover versus clover-on-HISQ ensembles. (iv) In both lattice formulations, variations in $r_{i}$ versus $m_{\pi}$ for fixed lattice spacing $a$, and versus $a$ for fixed $m_{\pi}$ are small. A detailed analysis is underway.

Acknowledgements: We thank the MILC Collaboration for the 2+1+1-flavor HISQ ensembles and JLab/W\&M collaboration for the $2+1$ clover lattices. Simulations were carried out on computer facilities of (i) Oak Ridge Leadership Computing Facility; (ii) the USQCD Collaboration; (iii) the National Energy Research Scientific Computing Center and (iv) Institutional Computing at Los Alamos National Lab. Work supported by the DOE and Los Alamos LDRD. 


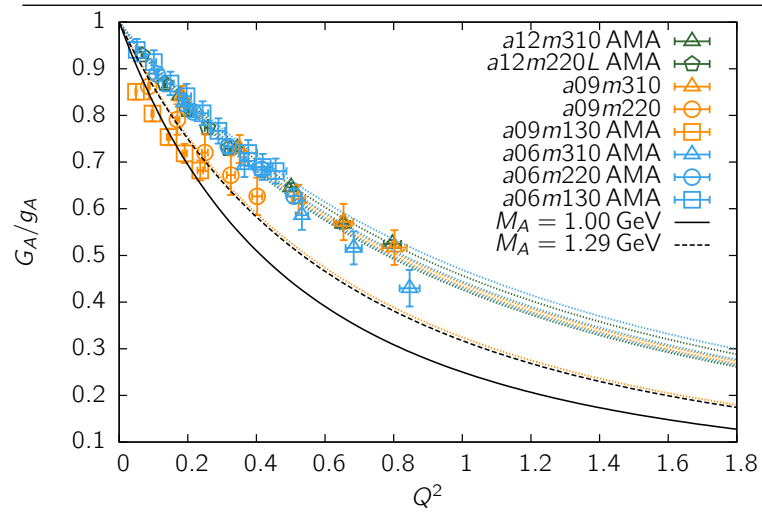

(a) $G_{A}$, Clover-on-HISQ analysis

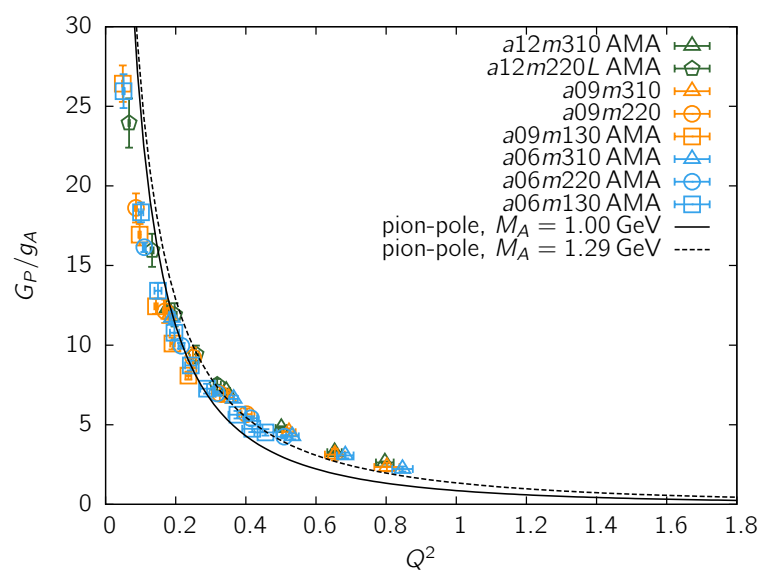

(c) $G_{P}$, Clover-on-HISQ analysis

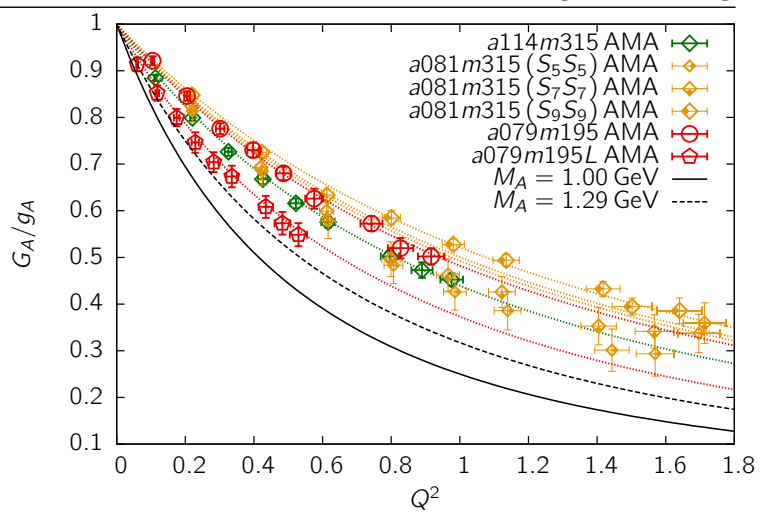

(b) $G_{A}$, Clover-on-Clover analysis

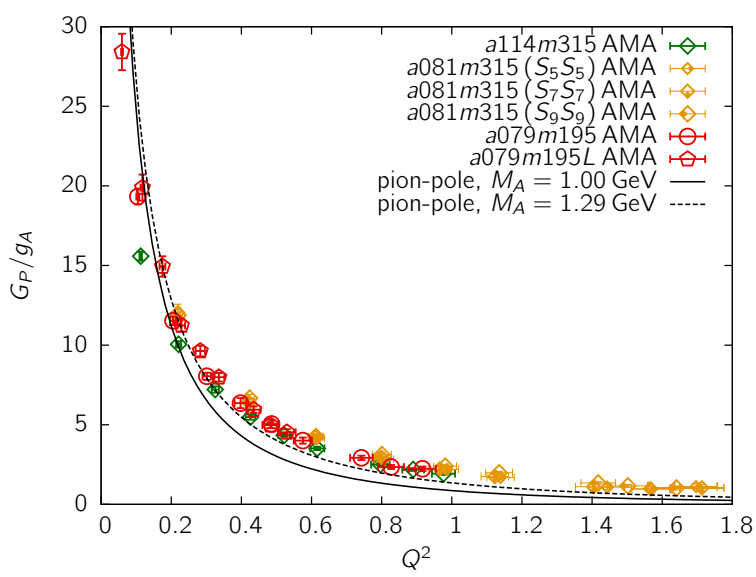

(d) $G_{P}$, Clover-on-Clover analysis

Figure 3: Data for $G_{A}\left(Q^{2}\right)$ and $G_{P}\left(Q^{2}\right)$ with dipole fits to $G_{A}\left(Q^{2}\right)$, pion-pole dominance to $G_{P}\left(Q^{2}\right)$.

\section{References}

[1] P. J. Mohr et al., CODATA group, Rev. Mod. Phys. 88 (2016), no. 3 035009, [1507. 07956].

[2] A. Antognini et al., EPJ Web Conf. 113 (2016) 01006, [1509. 03235].

[3] R. Pohl1 et al., CREMA, Science 353 (2016), no. 6300 669-673.

[4] I. T. Lorenz, H. W. Hammer, and U.-G. Meissner, Eur. Phys. J. A48 (2012) 151, [1205 . 6628].

[5] K. A. Olive et al., Particle Data Group, Chin. Phys. C38 (2014) 090001.

[6] B. Bhattacharya, R. J. Hill, and G. Paz, Phys. Rev. D84 (2011) 073006, [11 08 . 0423 ].

[7] A. S. Meyer et al., Phys. Rev. D93 (2016), no. 11 113015, [1603.03048].

[8] R. Gupta et al., PoS LATTICE2016 (2016) 157.

[9] T. Bhattacharya et al., Phys. Rev. D94 (2016), no. 5 054508, [1606.070 49].

[10] B. Yoon et al., 1611.07452.

[11] R. G. Sachs, Phys. Rev. 126 (1962) 2256-2260.

[12] G. S. Bali et al., Comput. Phys. Commun. 181 (2010) 1570-1583, [0 910 . 3970].

[13] T. Blum, T. Izubuchi, and E. Shintani, Phys. Rev. D88 (2013), no. 9 094503, [1208.4349].

[14] J. J. Kelly, Phys. Rev. C70 (2004) 068202.

[15] R. J. Hill and G. Paz, Phys. Rev. D82 (2010) 113005, [1008 . 4619]. 